\title{
La rehabilitación y fisioterapia domiciliaria en las prótesis de rodilla
}

\section{Home care rehabilitation and physiotherapy in knee prosthesis}

\author{
R. López-Liria ${ }^{1}$, F. A. Vega-Ramírez ${ }^{2}$, D. Catalán-Matamoros ${ }^{1}$, \\ D. Padilla-Góngora ${ }^{3}$, M. C. Martínez-Cortés ${ }^{3}$, A. Mesa-Ruiz ${ }^{4}$
}

\section{RESUMEN}

En esta revisión se analizan los estudios sobre rehabilitación y fisioterapia domiciliaria existentes en pacientes intervenidos con prótesis de rodilla, identificando y sintetizando los resultados de las intervenciones medidas en términos de fuerza muscular, función, amplitud articular, marcha, calidad de vida del paciente y coste del servicio, comparados con otras alternativas de tratamiento.

Se han revisado nueve bases de datos: IME, ENFISPO, CUIDEN PLUS, PUBMED, EMBASE, CINAHL, PEDro, COCHRANE library Plus y TRIP DATA BASE donde 18 estudios cumplieron los criterios de inclusión con información sobre 4.996 pacientes.

La rehabilitación domiciliaria varía de un lugar a otro y entre estudios (formas de atención, aplicación, duración, variables medidas); pero se respalda la idea de que ésta es tan efectiva y aceptable como la recibida ambulatoriamente. Acelera la consecución de logros funcionales a corto y medio plazo, conllevando estancias hospitalarias más breves y reducción de costes.

Palabras clave. Prótesis. Rodilla. Rehabilitación. Fisioterapia. Domicilio.

\begin{abstract}
This review analyzes the existing studies on home care rehabilitation and physiotherapy in patients with a knee prosthesis intervention, identifying and synthesising the results of the interventions measured in terms of muscular strength, function, extension of the joint, walking, patient's quality of life and cost of the service, compared with other alternative treatments.

Nine databases have been analysed: IME, ENFISPO, CUIDEN PLUS, PUBMED, EMBASE, CINAHL, PEDro, COCHRANE library Plus and TRIP DATA BASE where 18 studies met the criteria for inclusion with information on 4,996 patients.

Home care rehabilitation varies from one place to another and between studies (forms of care, application, duration, variable measures); but the idea is supported that this is as effective and acceptable as that received in an outpatients' department. It accelerates the attainment of functional achievements in the short and medium term, entailing very brief hospital stays and a reduction in costs.
\end{abstract}

Key words. Prosthesis. Knee. Rehabilitation. Physiotherapy. Home.
1. Facultad de Ciencias de la Salud. Universidad de Almería, Almería, España.

2. Servicio de Salud de Castilla La Mancha, Toledo, España.

3. Facultad de Ciencias de la Salud. Universidad de Almería, Almería, España.

4. Servicio Andaluz de Salud, Granada, España.

Recepción: 10 de octubre de 2011

Aceptación provisional: 12 de diciembre de 2011

Aceptación definitiva: 19 de diciembre de 2011

\section{Correspondencia:}

Remedios López Liria

Facultad de Ciencias de la Salud. Universidad de Almería

Crta. Sacramento S/N

04120 Almería

Correo electrónico: rll040@ual.es 


\section{INTRODUCCIÓN}

La edad aumenta la prevalencia de enfermedades degenerativas e incapacitantes, ello posee importantes implicaciones sanitarias, sociales, económicas y políticas ${ }^{1}$. La osteoartrosis es la enfermedad articular más frecuente, padeciéndola más del $70 \%$ de los mayores de 50 años. Se manifiesta mediante signos radiológicos degenerativos en el $5-15 \%$ de la población entre 35 y 74 años, siendo la causa más habitual de incapaci$\mathrm{dad}^{2}$. Según el estudio EPISER ${ }^{3}$, el $10,2 \%$ de los adultos presentan artrosis sintomática de rodilla, cifras similares a las encontradas en estudios de otros países. De este grupo el $25 \%$ podría ser susceptible de artroplastia ${ }^{4,5}$.

Los estudios epidemiológicos sobre artrosis en España ${ }^{6,7}$ reflejan que es un motivo de frecuente visita médica y elevado consumo de fármacos, existiendo poca difusión de medidas higiénicas de tratamiento como ejercicio y dietas. Hay un escaso empleo de ayudas técnicas y adaptaciones; suelen ser pacientes pluripatológicos que sufren una gran repercusión en su calidad de vida. Se da un elevado coste de recursos y la artroplastia total de rodilla es una solución en el tratamiento de la discapacidad, convirtiéndola en una de las actividades médicas más costo-efectivas ${ }^{8}$. En los últimos años se ha incrementado la creación de unidades funcionales de artroplastia de rodilla, disminuyendo significativamente la estancia media en el hospital y los costes del proceso, pero unido a un aumento importante de estas intervenciones ${ }^{9}$.

La rehabilitación adecuada es un requisito para el éxito de la prótesis ${ }^{10-12}$, devolviendo el rango articular restringido tras la intervención y la funcionalidad para las actividades de la vida diaria.

Existen diversas formas de tratamiento una vez que el paciente es intervenido de artroplastia de rodilla: en unidades de rehabilitación para procesos subagudos; fisioterapia ambulatoria; a domicilio, asistidos por fisioterapeutas; sólo asesorados por folletos con ejercicios; rehabilitación multidisciplinaria ${ }^{13}$, etc. Por ello, aún hay controversias sobre cuál es la forma más efectiva para su aplicación. Algunos autores recomiendan la rehabilitación domiciliaria en comparación con la rehabilitación habitual, incluso después del alta precoz hospitalaria ${ }^{14,15}$. Sin embargo, otros exponen que los beneficios son mínimos o similares con respecto al tratamiento ambulatorio ${ }^{16,17}$.

En los últimos años las políticas sanitarias para mantener al paciente en su domicilio se han incrementado, avanzando en la accesibilidad de los servicios sanitarios para las personas con mayor dificultad y disminuyendo los costes asociados a la hospitalización ${ }^{18,19}$.

La consideración de nuevas alternativas de tratamientos de rehabilitación como los ofrecidos en el domicilio por el sistema sanitario público ${ }^{20}$, da como resultado la necesidad de desarrollar estudios para demostrar cuáles son los tratamientos más eficaces. Al mismo tiempo surgen cuestiones que investigar como valorar la repercusión que están teniendo los programas de rehabilitación domiciliaria en políticas y prácticas sociosanitarias.

El objetivo de esta investigación ha sido analizar los estudios sobre rehabilitación y fisioterapia domiciliaria existentes en pacientes intervenidos con prótesis de rodilla, identificando y sintetizando los resultados de las intervenciones medidas en términos de fuerza muscular, función, amplitud articular, marcha, calidad de vida del paciente y coste del servicio, comparados con otras alternativas de tratamiento.

Se especificó el problema y se definió la pregunta clínica sobre ¿qué evidencia existe en cuanto a la efectividad de la rehabilitación o fisioterapia domiciliaria como tratamiento comparada con otras intervenciones, para la mejora de los pacientes con prótesis de rodilla?

\section{MATERIAL Y MÉTODOS}

Se ha llevado a cabo una revisión de la literatura tomando como criterio las fases que especifican Clarke y Oxman ${ }^{21}$.

La población diana seleccionada fue la intervenida de prótesis de rodilla. Los criterios de inclusión, los siguientes:

a) Artículos obtenidos a partir de los descriptores definidos para la búsqueda en español: Rehabilitación; Fisioterapia; 
Domicilio; Artroplastia; Prótesis; Rodilla. Los descriptores en inglés fueron: Physical therapy modalities; Physical therapy techniques; Physical therapy; Physiotherapy; Rehabilitation; Exercise therapy; Terapeutic exercise; Home care services; Home care; Home care servided hospital based; Rehabilititation at home; Homerehabilitation; Home physical therapy; Implants; Arthroplasty; Replacement; knee Prótesis; knee. b) Estudios de diseño observacional, experimental o cuasiexperimental realizados en pacientes con prótesis de rodilla que recibiesen rehabilitación o fisioterapia domiciliaria en comparación con la atención rehabilitadora convencional, fisioterapia ambulatoria u otros cuidados estandarizados (que precisasen o no, una estructura organizativa y de personal). También se consideraron aquellas revisiones sistemáticas, meta-análisis o revisiones bibliográficas.

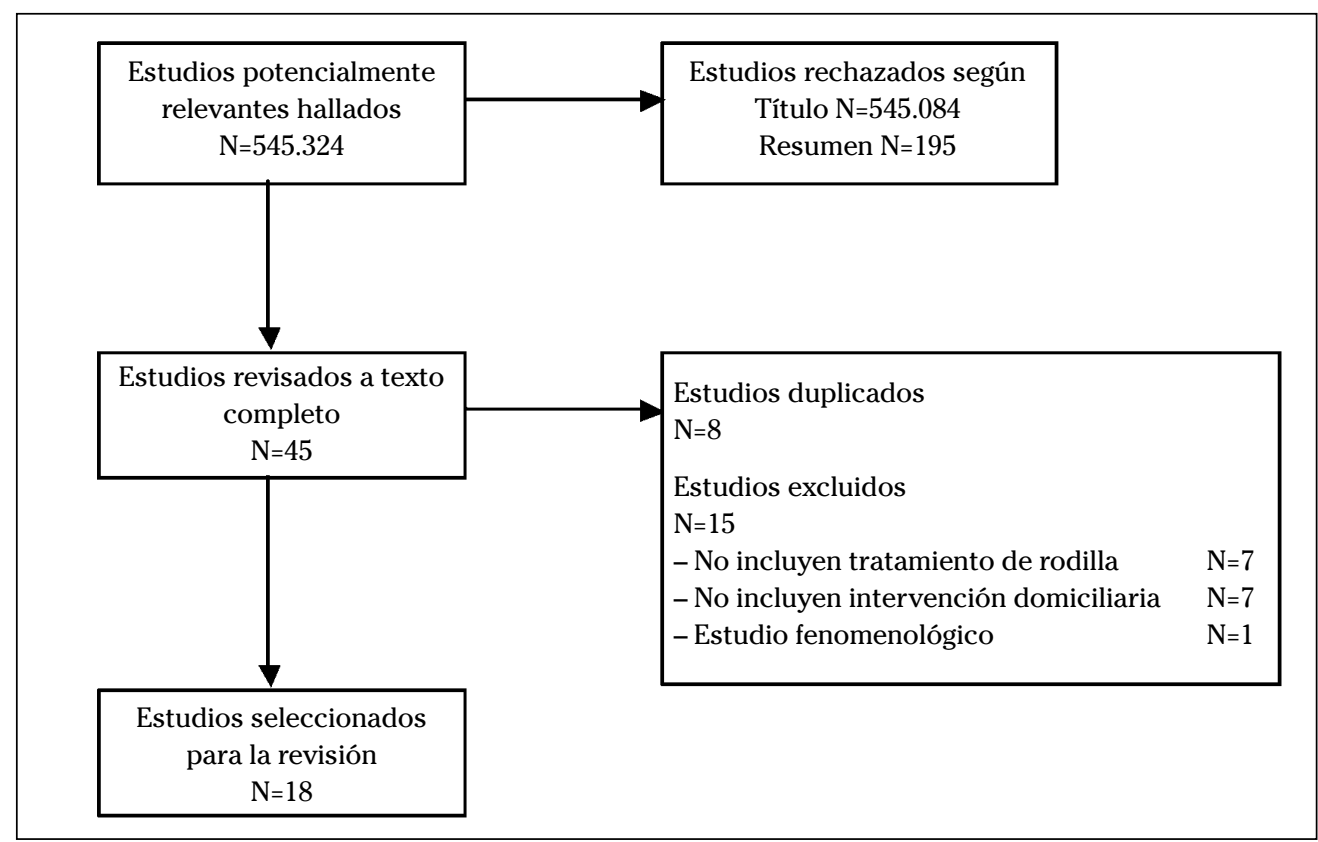

Figura 1. Etapas seguidas para la obtención de los artículos seleccionados

Las variables analizadas han sido:

- Características del estudio (diseño, muestra).

- Características del programa de rehabilitación o fisioterapia y tratamiento a comparar.

- Medidas de resultado consideradas en los distintos estudios (dolor, nivel de actividad o funcionalidad, rango articular, fuerza muscular, marcha, calidad de vida, satisfacción, duración de la estancia hospitalaria, rein- gresos, utilización de servicios, coste o coste-efectividad, duración del tratamiento y cantidad de sesiones).

- Efectividad de la rehabilitación o fisioterapia domiciliaria comparada con otros tratamientos.

c) Artículos o trabajos de investigación que hubiesen sido publicados en el periodo entre el 1 de enero 2000 y enero de 2009.

La elección de las palabras clave/descriptores para realizar la búsqueda de información en inglés, se realizó mayorita- 
riamente a través del MeSH Database de PubMed. En relación a los operadores booleanos, fueron utilizados AND y OR.

Las Bases de Datos nacionales e internacionales seleccionadas para la realización de la búsqueda fueron IME, ENFISPO, CUIDEN PLUS, PUBMED, EMBASE, CINAHL, PEDro, COCHRANE library Plus y TRIP DATA BASE.

Posteriormente dos investigadores revisaron de forma independiente todos los resúmenes y los títulos de los estudios identificados mediante la estrategia de búsqueda; y en base a los criterios de selección y los objetivos, obtenidos los artículos potencialmente apropiados, se evaluaron de forma independiente cada uno de for- ma completa y si no se alcanzaba ningún consenso acerca de la inclusión/exclusión posible, un tercer investigador realizó la mediación.

Los resultados obtenidos en la búsqueda, así como los artículos seleccionados se muestran en la figura 1 . La tabla 1 se presenta como ejemplo del recorrido realizado hasta la selección final de estudios, en tres de las bases de datos internacionales.

Se identificaron 33 estudios en la primera revisión de los artículos, pero una vez analizados los textos completos el número se redujo a 18, ya que todos no se habían ajustado a los objetivos planteados en esta investigación.

Tabla 1. Resultados de la revisión bibliográfica en 3 bases de datos internacionales

\begin{tabular}{|c|c|c|}
\hline Bases de datos internacionales: descriptores & Resultados & Selección ${ }^{\text {a, b }}$ \\
\hline $\begin{array}{l}\text { PUBMED } \\
\text { 1. "Physical Therapy Modalities" or "Physical Therapy } \\
\text { (Specialty) or "Rehabilitation" or "Exercise Therapy" } \\
\text { 2. "Home care services" or "Home care services, hospital-based" } \\
\text { 3. \#1 and \#2 } \\
\text { 4. "Arthroplasty, Replacement, Knee" or "knee prosthesis" or } \\
\text { "Prostheses and implants" or "Arthroplasty" } \\
\text { 5. \#1 and \#2 and \#4 }\end{array}$ & $\begin{array}{r}178.791 \\
33.396 \\
1.041 \\
56.976 \\
20\end{array}$ & $\begin{array}{r}(\text { Galea, 2008) })^{22} \\
\text { (Sigurdsson, 2008) }{ }^{23} \\
(\text { Chimenti, 2007) } \\
(\text { Collins, 2007) } \\
(\text { Iyengar, 2007) } \\
{ }^{25} \\
\text { (Van den Akker-Scheek, 2007) })^{26} \\
(\text { Polzien, 2006) })^{27} \\
(\text { Lavernia, 2006) } \\
(\text { Mitchell, 2005) })^{14} \\
(\text { De Pablo, 2004) })^{28} \\
(\text { Kramer, 2003) })^{29} \\
(\text { Loft, 2003) })^{30} \\
(\text { Engh, 2001) })^{31} \\
(\text { Heaton, 2000) })^{32} \\
(\text { Kane, 2000) })^{33} \\
(\text { Woo, 2000) })^{34}\end{array}$ \\
\hline $\begin{array}{l}\text { EMBASE } \\
\text { 1. "Physiotherapy" or "rehabilitation" or "kinesiotherapy" } \\
\text { 2. "Home Physiotherapy" or "home rehabilitation" or "home } \\
\text { care" } \\
\text { 3. \#1 and \#2 } \\
\text { 4. "Knee arthroplasty" or "total knee replacement" or "knee } \\
\text { prosthesis" } \\
\text { 5. \#3 and \#4 }\end{array}$ & $\begin{array}{r}192.298 \\
40.615 \\
2.323 \\
\\
16.022 \\
20\end{array}$ & $\begin{array}{l}\text { (Mahomed, 2008) })^{35} \\
(\text { Khan, 2008) } \\
{ }^{36} \\
(\text { Lenssen, 2008) } \\
(\text { Collins, 2007) } \\
(\text { Chimenti, 2007) } \\
\left(^{15}\right. \\
(\text { Mitchell, 2005) })^{14} \\
(\text { Kramer, 2003) } \\
\quad(\text { Frost, 2002) }\end{array}$ \\
\hline $\begin{array}{l}\text { PEDro } \\
\text { Physical Therapy and lower leg or knee } \\
\text { Physiotherapy and lower leg or knee } \\
\text { Home Physiotherapy and lower leg or knee } \\
\text { Rehabilitation and lower leg or knee } \\
\text { Home rehabilitation and lower leg or knee } \\
\text { Arthroplasty and lower leg or knee }\end{array}$ & $\begin{array}{r}105 \\
77 \\
10 \\
167 \\
33 \\
47\end{array}$ & $\begin{array}{l}(\text { Khan, 2008) } \\
\left(^{36}\right. \\
(\text { Menssen, 2008) })^{11} \\
\left(\text { Mitchell, 2005) }{ }^{14}\right. \\
(\text { Moffet, 2004) } \\
(\text { Kramer, 2003) } \\
{ }^{29} \\
\left(\text { Davies, 2003) }{ }^{39}\right. \\
(\text { Frost, 2002) }\end{array}$ \\
\hline
\end{tabular}

a Ordenadas por fecha de publicación; ${ }^{\mathrm{b}}$ Primer autor, año de publicación

' $\mathrm{Se}$ han omitido aquellos descriptores con cero resultados 


\section{RESULTADOS}

Los ensayos y revisiones incluidas contenían información de un total de 4.996 pacientes donde se identificaron sus características, la atención recibida tras ser intervenidos y la duración del seguimiento, las medidas de resultado y los efectos del servicio (información resumida en la tabla 2).

A continuación se expone el análisis de contenido de los estudios seleccionados, según las variables incluidas.

\section{Diseño de los estudios}

El 22,3\% de las investigaciones fueron de diseño descriptivo (4), los estudios experimentales o pseudo-experimentales fueron el $61 \%$ (11) y el $16,7 \%$ revisiones sistemáticas o meta-análisis (3).

La moda de las muestras seleccionadas en los estudios fue de 160 pacientes. Con una media de 133 pacientes (sin incluir los dos extremos, donde el máximo fue 1.034 pacientes ${ }^{25}$ y el mínimo 47 pacientes ${ }^{37}$; y las tres revisiones sistemáticas rondaron entre los 600 y 1.000 pacientes).

\section{Características de los programas de rehabilitación/tratamientos}

La búsqueda bibliográfica halló tres revisiones sistemáticas cada una de las cuales trataron aspectos diferentes del tema de la rehabilitación domiciliaria: Khan y $\mathrm{col}^{36}$ seleccionan cinco estudios, donde en dos de ellos se aborda la rehabilitación hospitalaria y tres la domiciliaria; Milne y $\mathrm{col}^{41}$ indagan en el tema del movimiento pasivo continuo más fisioterapia y distintos tratamientos que no incluían este tipo de movilización, entre ellos la fisioterapia domiciliaria. Minns y coll $^{42}$ evalúan la efectividad de la fisioterapia donde unas veces se incluían los ejercicios en casa, la habitual del hospital u otros tipos de intervenciones fisioterapéuticas a comparar.

Cinco ensayos ${ }^{14,15,28,25,35}$ trataron el tema de la rehabilitación domiciliaria versus ambulatoria. El estudio de Mitchell y col $^{14}$ explica exhaustivamente el procedimiento llevado a cabo durante las sesiones de fisioterapia del preoperatorio y del postoperatorio.
Se comparó la rehabilitación ambulatoria u hospitalaria con un protocolo domiciliario en cinco de los estudios seleccionados. Tres de ellos ${ }^{16,40,42}$ entregaban el protocolo de rehabilitación mediante un folleto descriptivo de los ejercicios, aunque no todos los estudios especificaron el programa ${ }^{16}$. Brunenberg y col ${ }^{43}$ se refieren a los cuidados con sólo información ofrecida sobre el alta durante el periodo de admisión. Otros autores como Moffet y col $^{35}$ especifican minuciosamente el tratamiento; sus dos grupos de pacientes estuvieron divididos entre los que realizaron rehabilitación supervisada y ejercicios en casa (experimental), con un grupo control que recibieron cuidados estándar. Kramer y col ${ }^{29}$ comparan un programa de rehabilitación ambulatoria de 12 semanas (unas 15 sesiones) y los ejercicios en casa controlados telefónicamente por un fisioterapeuta (con cinco llamadas) y la revisión por el cirujano.

Otro estudio ${ }^{11}$ comparó la rehabilitación domiciliaria y el movimiento pasivo continuo con la rehabilitación ambulatoria.

Dos ensayos ${ }^{24,44}$ trataron la rehabilitación domiciliaria comparada con la realizada en un servicio hospitalario de subagudos antes de continuar el tratamiento en el hogar. Nelly y col ${ }^{44}$ describieron detalladamente ambos programas en casa y en el hospital.

Van den Akker-Scheek y col $^{26}$ enfatizaron la continuación de la fisioterapia domiciliaria a través de vídeos, cartas y consultas telefónicas (Programa Groningen Orthopaedic Exit Strategy) comparándolo con un grupo que sólo recibió instrucciones durante el ingreso para la intervención y una llamada tras el alta.

Y Frost y col ${ }^{37}$ en 2004, mostraron interés en averiguar si es más eficaz un programa tradicional de ejercicios en casa o un programa funcional de fisioterapia llevado a cabo en el domicilio.

\section{Medidas de resultado}

El dolor fue una variable que consideraron nueve de los 18 estudios tenidos en cuenta. Unas veces medido a través de la escala $\mathrm{EVA}^{16}$, la medicación usada ${ }^{11,44}$, junto a la inflamación ${ }^{41}$ o con otros procedimien$\operatorname{tos}^{17,24,26,29,37}$. 
Tabla 2. Descripción de los artículos incluidos en la revisión a

\begin{tabular}{|c|c|c|c|c|}
\hline $\begin{array}{l}\text { Autores } \\
\text { y Año }{ }^{b}\end{array}$ & Diseño o Método & Participantes & Intervenciones y Seguimiento & Variables medidas y Resultados \\
\hline $\begin{array}{l}\text { Mozo } \\
\mathrm{y} \mathrm{col}^{16} \\
2008\end{array}$ & $\begin{array}{l}\text { Estudio prospectivo, } \\
\text { no aleatorizado. }\end{array}$ & $\begin{array}{l}122 \text { pacientes } \\
-40 \text { en programa } \\
\text { RHB }^{\mathrm{c}} \text { domiciliario } \\
\text { (protocolo entregado) } \\
-82 \text { pacientes } \\
\text { con tratamiento } \\
\text { ambulatorio } \\
\text { (ejercicios activo } \\
\text { asistidos). }\end{array}$ & $\begin{array}{l}\text { Comparar los resultados del pro- } \\
\text { tocolo entregado en papel a los } \\
\text { pacientes durante los primeros } 45 \\
\text { días tras la intervención de ATR d; } \\
\text { con los ejercicios activo asistidos } \\
\text { del tratamiento ambulatorio duran- } \\
\text { te } 5 \text { días a la semana; una media de } \\
12 \text { sesiones. } \\
\text { Seguimiento en el preoperatorio, } 8^{\circ} \\
\text { día y } 6^{0} \text { mes. }\end{array}$ & $\begin{array}{l}\text { Se estudió el dolor (EVA), funcionalidad, } \\
\text { Indice de Barthel, capacidad de marcha y } \\
\text { calidad de vida (SF-36). } \\
\text { Sólo hubo diferencias en la medida del ran- } \\
\text { go articular al } 8^{\circ} \text { y } 6^{0} \text { mes. } \\
\text { La rehabilitación domiciliaria ofrecía resul- } \\
\text { tados similares a los de la rehabilitación } \\
\text { ambulatoria en los pacientes selecciona- } \\
\text { dos. }\end{array}$ \\
\hline $\begin{array}{l}\text { Khan } \\
\mathrm{y} \mathrm{col}^{36} \\
2008\end{array}$ & $\begin{array}{l}\text { Revisión sistemática } \\
\text { Cochrane. Búsqueda } \\
\text { electrónica en } \\
\text { MEDLINE, EMBASE } \\
\text { Y CINAHL, hasta } \\
\text { septiembre de } 2006 . \\
\text { Ensayos controlados } \\
\text { aleatorios. } \\
\text { Los ensayos de } \\
\text { baja calidad debido } \\
\text { a la ausencia de } \\
\text { cegamiento. }\end{array}$ & 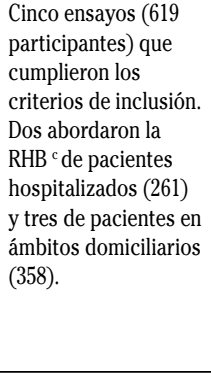 & $\begin{array}{l}\text { Evaluar las pruebas para la efecti- } \\
\text { vidad de la RHB }{ }^{\mathrm{c}} \text { multidisciplinaria } \\
\text { tras ATR }{ }^{\mathrm{d}} \text { o cadera. } \\
\text { Seguimiento a los } 6 \text { meses. }\end{array}$ & $\begin{array}{l}\text { Las medidas de resultado fueron la limita- } \\
\text { ción de la deficiencia y la actividad/función; } \\
\text { costo de la atención, duración de la estan- } \\
\text { cia hospitalaria, utilización de servicios, } \\
\text { readmisión, tasas de mortalidad y carga del } \\
\text { cuidador. } \\
\text { La atención multidisciplinaria domiciliaria } \\
\text { mejoró la ganancia funcional, la calidad de } \\
\text { vida y redujo la estancia hospitalaria a me- } \\
\text { dio plazo (6 meses). }\end{array}$ \\
\hline $\begin{array}{l}\text { Mahomed y } \\
\mathrm{col}^{35}, 2008\end{array}$ & $\begin{array}{l}\text { Ensayo clínico } \\
\text { aleatorizado. }\end{array}$ & 234 pacientes & $\begin{array}{l}\text { Evaluar la efectividad y coste de la } \\
\text { rehabilitación en casa ( } 8 \text { sesiones) } \\
\text { comparada con la fisioterapia am- } \\
\text { bulatoria ( } 17 \text { sesiones) después de } \\
\text { ATR }{ }^{\mathrm{d}} \text { o cadera. } \\
\text { La evaluación fue previa a la inter- } \\
\text { vención, a los } 3 \text { y a los } 12 \text { meses tras } \\
\text { la artroplastia. }\end{array}$ & $\begin{array}{l}\text { Los resultados medidos fueron la escala } \\
\text { WOMAC, cuestionario de calidad de vida } \\
\text { (SF-36) y satisfacción del paciente. } \\
\text { Los dos grupos mostraron una sustancial } \\
\text { mejora a los } 3 \text { y a los } 12 \text { meses, pero sin di- } \\
\text { ferencias estadísticamente significativas ni } \\
\text { en el escala WOMAC, SF-36 o satisfacción. } \\
\text { Los costes fueron menores en el domicilio. }\end{array}$ \\
\hline $\begin{array}{l}\text { Mockford y } \\
\mathrm{col}^{40}, 2008\end{array}$ & $\begin{array}{l}\text { Ensayo clínico } \\
\text { aleatorizado } \\
\text { prospectivo. Doble } \\
\text { ciego }\end{array}$ & $\begin{array}{l}150 \text { participantes } \\
\text { Grupo A: } 71 \\
\text { Grupo B: } 72 \\
\text { Pérdidas: } 7\end{array}$ & $\begin{array}{l}\text { Ambos grupos recibieron trata- } \\
\text { miento durante la hospitalización. } \\
\text { Grupo A: Tratamiento fisioterápi- } \\
\text { co durante } 6 \text { semanas (media de } 7 \\
\text { sesiones) } \\
\text { Grupo B: ningún tratamiento. } \\
\text { Todos los pacientes recibieron un } \\
\text { régimen de ejercicios para realizar } \\
\text { en casa. } \\
\text { Medidas tomadas preoperatoria- } \\
\text { mente, a los } 3 \text { meses y al año de la } \\
\text { intervención. }\end{array}$ & $\begin{array}{l}\text { Se midió el rango articular, the Oxford Knee } \\
\text { Store, el Barlett patellar store y la calidad } \\
\text { de vida con el SF-12. } \\
\text { Aunque los pacientes en tratamiento obtu- } \\
\text { vieron un mayor rango articular, la diferen- } \\
\text { cia no fue estadísticamente significativa. A } \\
\text { los } 3 \text { meses los pacientes con tratamiento } \\
\text { mejoraron más rápidamente, no hubo dife- } \\
\text { rencias al año. }\end{array}$ \\
\hline $\begin{array}{l}\text { Lenssen y } \\
\operatorname{col}^{11}, 2008\end{array}$ & $\begin{array}{l}\text { Ensayo clínico } \\
\text { aleatorizado con } \\
\text { cegamiento en } \\
\text { la asignación del } \\
\text { tratamiento }\end{array}$ & $\begin{array}{l}60 \text { pacientes en total. } \\
30 \text { pacientes en el } \\
\text { grupo experimental y } \\
30 \text { en el control. }\end{array}$ & $\begin{array}{l}\text { Grupo experimental: recibió movi- } \\
\text { miento pasivo continuo (MPC) y } \\
\text { fisioterapia en casa durante } 17 \text { días } \\
\text { consecutivos. } \\
\text { Grupo control el mismo tratamien- } \\
\text { to los } 4 \text { días de hospitalización y } \\
\text { luego sólo fisioterapia durante las } 2 \\
\text { primeras semanas. } \\
\text { Medidas tomadas al final del tra- } \\
\text { tamiento, } 17 \text { días después de la in- } \\
\text { tervención; a las } 6 \text { semanas y a los } \\
\text { tres meses. }\end{array}$ & $\begin{array}{l}\text { Las medidas consideradas fueron el rango } \\
\text { articular de movilidad (goniómetro) y la } \\
\text { funcionalidad (WOMAC), efecto percibido, } \\
\text { medicación usada para el dolor, satisfac- } \\
\text { ción, adherencia al tratamiento, duración } \\
\text { del tratamiento y cantidad. } \\
\text { Como conclusiones exponen que el uso } \\
\text { prolongado del movimiento pasivo conti- } \\
\text { nuo tiene pequeños y cortos efectos en el } \\
\text { rango articular conseguido. }\end{array}$ \\
\hline
\end{tabular}




\begin{tabular}{|c|c|c|c|c|}
\hline $\begin{array}{l}\text { Autores } \\
\text { y Año }^{\text {b }}\end{array}$ & Diseño o Método & Participantes & Intervenciones y Seguimiento & Variables medidas y Resultados \\
\hline $\begin{array}{l}\text { Milne y } \\
\mathrm{col}^{41}, 2008\end{array}$ & $\begin{array}{l}\text { Revisión sistemática } \\
\text { Cochrane. Búsqueda } \\
\text { electrónica en } \\
\text { MEDLINE, Embase, } \\
\text { CINAHL, HEALTH } \\
\text { STAR y CURRENT } \\
\text { CONTENTS para } \\
\text { identificar ensayos } \\
\text { controlados } \\
\text { aleatorios. La calidad } \\
\text { metodológica fue de } \\
\text { dos de cinco. }\end{array}$ & $\begin{array}{l}14 \text { ensayos (952 } \\
\text { participantes) }\end{array}$ & $\begin{array}{l}\text { Grupo experimental: fisioterapia y } \\
\text { MPC } \\
\text { Grupo control: fisioterapia (en dis- } \\
\text { tintos ámbitos) } \\
\text { Evaluar la efectividad del movi- } \\
\text { miento pasivo continuo tras ATR }\end{array}$ & $\begin{array}{l}\text { Se midió la amplitud de movimiento pasivo } \\
\text { y activo de la rodilla, duración de la estan- } \\
\text { cia hospitalaria, dolor, inflamación, fuerza } \\
\text { del cuádriceps. } \\
\text { Se encontraron resultados que favorecían } \\
\text { al MPC en la comparación con la fisiotera- } \\
\text { pia sola al final del tratamiento. Incrementó } \\
\text { de una manera estadísticamente significati- } \\
\text { va la flexión activa de rodilla y disminuyó la } \\
\text { estancia hospitalaria. }\end{array}$ \\
\hline $\begin{array}{l}\text { Collins y } \\
\text { coll }^{15}, 2007\end{array}$ & $\begin{array}{l}\text { Estudio descriptivo } \\
\text { retrospectivo. }\end{array}$ & $\begin{array}{l}17 \text { expedientes pre- } \\
\text { prótesis de rodilla } \\
\text { con } 39 \text { post-prótesis } \\
\text { de rodilla }\end{array}$ & $\begin{array}{l}\text { Determinar si el post prótesis afec- } \\
\text { ta al número de visitas en casa para } \\
\text { los pacientes de Medicare, a la dis- } \\
\text { tancia recorrida por el paciente, los } \\
\text { cambios en el recorrido articular de } \\
\text { la rodilla, y con qué frecuencia fue } \\
\text { recomendada la fisioterapia domi- } \\
\text { ciliaria con respecto a los que eran } \\
\text { tratados en el hospital. }\end{array}$ & $\begin{array}{l}\text { Se midió el número de visitas de fisiotera- } \\
\text { pia, la distancia de deambulación, el rango } \\
\text { articular para la flexión y extensión, los dis- } \\
\text { positivos de apoyo a la marcha y las reco- } \\
\text { mendaciones de fisioterapia ambulatoria. } \\
\text { El número de visitas a domicilio decreció } \\
\text { después de la implementación de la pró- } \\
\text { tesis. } \\
\text { En cambio, la distancia de deambulación } \\
\text { fue mayor después de la intervención. } \\
\text { No hubo grandes diferencias en la mejoría } \\
\text { del rango articular (ya que las visitas de fisio- } \\
\text { terapia domiciliaria fueron más funcionales). }\end{array}$ \\
\hline $\begin{array}{l}\text { Minns y } \\
\operatorname{col}^{42}, 2007\end{array}$ & $\begin{array}{l}\text { Revisión sistemática, } \\
\text { búsqueda electrónica } \\
\text { en AMED, CINAHL, } \\
\text { Embase, MEDLINE, } \\
\text { Cochrane, PEDro, } \\
\text { Department of Health } \\
\text { National Research } \\
\text { Register } \\
\text { Estudios randomizados } \\
\text { controlados. }\end{array}$ & $\begin{array}{l}614 \text { pacientes, } \\
\text { de los } 6 \text { estudios } \\
\text { identificados y } 5 \\
\text { incluidos. }\end{array}$ & $\begin{array}{l}\text { Evaluar la efectividad de la fisiotera- } \\
\text { pia después de prótesis de rodilla. } \\
\text { Se incluían programas de ejercicios } \\
\text { de fisioterapia, ambulatoriamente, } \\
\text { en la comunidad o en casa (ejerci- } \\
\text { cios de estiramiento e isométricos } \\
\text { de cuádriceps aprendidos durante } \\
\text { la estancia hospitalaria). }\end{array}$ & $\begin{array}{l}\text { Midieron las actividades de la vida diaria, } \\
\text { marcha, calidad de vida, fuerza muscular, } \\
\text { rango articular, calidad de los estudios. } \\
\text { Hallan diferencias a favor de los ejercicios } \\
\text { funcionales en pacientes que realizan fisio- } \\
\text { terapia después de ATRd a los } 3-4 \text { meses del } \\
\text { postoperatorio, también en el rango articu- } \\
\text { lar y la calidad de vida. Pero los beneficios } \\
\text { no mostraron evidencia más allá de un año. }\end{array}$ \\
\hline $\begin{array}{l}\text { Chimenti } \\
\text { e Ingersoll } \\
\mathrm{col}^{24}, 2007\end{array}$ & $\begin{array}{l}\text { Estudio descriptivo } \\
\text { prospectivo } \\
\text { longitudinal. }\end{array}$ & $\begin{array}{l}212 \text { pacientes } \\
\text { consecutivos } \\
\text { derivados a } \\
\text { fisioterapia tras } \\
\text { prótesis de rodilla. } \\
\text { - Fisioterapia } \\
\text { domiciliaria } \\
\mathrm{N}=74 \\
\text { - Unidad de } \\
\text { rehabilitación, } \mathrm{N}=138 \\
\text { pacientes }\end{array}$ & $\begin{array}{l}\text { Compara los episodios de cuidados } \\
\text { y resultados funcionales de los pa- } \\
\text { cientes derivados directamente a } \\
\text { casa para realizar fisioterapia de } \\
\text { aquellos derivados a una unidad de } \\
\text { rehabilitación subaguda antes de } \\
\text { pasar a fisioterapia en casa. } \\
\text { Seguimiento al finalizar el trata- } \\
\text { miento, al mes y a los tres meses de } \\
\text { la intervención. }\end{array}$ & $\begin{array}{l}\text { Las variables medidas son los cambios } \\
\text { físicos del paciente, dolor, flexión activa y } \\
\text { extensión de la rodilla, también pasiva, uso } \\
\text { de ayudas técnicas y fuerza muscular. } \\
\text { Como resultados se sugiere que la descarga } \\
\text { directa a casa tras artroplastia de rodilla es } \\
\text { una opción viable para muchos pacientes. }\end{array}$ \\
\hline $\begin{array}{l}\text { Van den } \\
\text { Akker- } \\
\text { Scheek y } \\
\mathrm{col}^{26}, 2007\end{array}$ & $\begin{array}{l}\text { Ensayo clínico } \\
\text { aleatorizado. Doble } \\
\text { ciego. }\end{array}$ & $\begin{array}{l}103 \text { pacientes } \\
\text { Grupo experimental: } \\
50 \text { pacientes } \\
\text { Grupo control: } 53 \\
\text { pacientes }\end{array}$ & $\begin{array}{l}\text { En el grupo experimental se enfa- } \\
\text { tiza la participación en la RHBc en } \\
\text { casa a través de vídeos, cartas y } \\
\text { consultas telefónicas. 2 veces al } \\
\text { mes en grupos de } 3-4 \text { pacientes du- } \\
\text { rante los } 6 \text { primeros meses. } \\
\text { En el control, sólo instrucciones } \\
\text { por parte del fisioterapeuta y una } \\
\text { llamada después del alta. } \\
\text { Validar el programa, que pretende } \\
\text { mejorar la RHBc tras ATR } \text { y cadera. } \\
\text { Seguimiento a las } 6 \text { y } 26 \text { semanas } \\
\text { tras la intervención. }\end{array}$ & $\begin{array}{l}\text { Se miden la eficacia, soporte social, dolor, } \\
\text { funcionalidad, actividad física, calidad de } \\
\text { vida, características del paciente, eficacia } \\
\text { de la } \mathrm{RHB}^{c} \text {, creencias, escala WOMAC. } \\
\text { No hubo diferencias entre los dos grupos } \\
\text { de pacientes en términos de cambios en } \\
\text { las medidas tomadas en el preoperatorio y } \\
\text { postoperatorio. Tampoco hubo diferencias } \\
\text { respecto al efecto del tratamiento ni a las } 6 \text {, } \\
\text { ni } 26 \text { semanas. } \\
\text { Como conclusiones, resumen que este tra- } \\
\text { tamiento necesita ser revisado. }\end{array}$ \\
\hline
\end{tabular}




\begin{tabular}{|c|c|c|c|c|}
\hline $\begin{array}{l}\text { Autores } \\
\text { y Año }\end{array}$ & Diseño o Método & Participantes & Intervenciones y Seguimiento & Variables medidas y Resultados \\
\hline $\begin{array}{l}\text { Iyengar y } \\
\operatorname{col}^{25}, 2007\end{array}$ & $\begin{array}{l}\text { Estudio Comparativo } \\
\text { con grupo control } \\
\text { histórico. No } \\
\text { está claro si fue } \\
\text { prospectivo o } \\
\text { retrospectivo; ni } \\
\text { el seguimiento } \\
\text { realizado. }\end{array}$ & $\begin{array}{l}1034 \text { pacientes } \\
\text { intervenidos de } \\
\text { prótesis de rodilla o } \\
\text { cadera. } \\
394 \text { pacientes } \\
\text { elegidos para la RHBc } \\
\text { a domicilio. } \\
\text { No se dio información } \\
\text { sobre el grupo } \\
\text { control. }\end{array}$ & $\begin{array}{l}\text { La hipótesis fue que la rehabili- } \\
\text { tación en casa podía disminuir la } \\
\text { permanencia en el hospital sin ma- } \\
\text { yores complicaciones ni reingresos, } \\
\text { comparada con la rehabilitación } \\
\text { convencional en el hospital. }\end{array}$ & $\begin{array}{l}\text { Analiza el coste efectividad. Número de } \\
\text { días en el hospital, en el tratamiento, núme- } \\
\text { ro de complicaciones y reingresos (para los } \\
\text { pacientes tratados en el domicilio). } \\
\text { Concluyen que el programa de rehabi- } \\
\text { litación domiciliaria reduce la estancia } \\
\text { hospitalaria en comparación con la RHB } \\
\text { convencional. No se observaron muchas } \\
\text { complicaciones ni reingresos. No se revela } \\
\text { una síntesis de los costes y beneficios del } \\
\text { servicio ofrecido. }\end{array}$ \\
\hline $\begin{array}{l}\text { Lavernia y } \\
\operatorname{col}^{17}, 2006\end{array}$ & $\begin{array}{l}\text { Estudio de Cohorte } \\
\text { Prospectivo }\end{array}$ & $\begin{array}{l}136 \text { pacientes con } \\
\text { ATRd de rodilla y } \\
\text { cadera. } \\
\text { No especifica el tipo } \\
\text { de servicio que se da } \\
\text { de rehabilitación en } \\
\text { cada grupo. }\end{array}$ & $\begin{array}{l}\text { Identificar el coste después de la } \\
\text { prótesis y estimar los gastos anua- } \\
\text { les de los servicios en una unidad } \\
\text { de rehabilitación con respecto a la } \\
\text { descarga en el hogar (Medicare). } \\
\text { Se toman medidas preoperatorias } \\
\text { y postoperatorias (media de } 8,6 \\
\text { meses). }\end{array}$ & $\begin{array}{l}\text { Gastos quirúrgicos, resultados en calidad } \\
\text { de vida, dolor, WOMAC; y el cuestionario } \\
\text { de bienestar, gastos directos e indirectos } \\
\text { (personal, cuidados de casa). } \\
\text { Los costes fueron superiores en las unida- } \\
\text { des de rehabilitación que en los hogares. } \\
\text { La mejoría en la función según la escala } \\
\text { WOMAC y en el dolor, se producía en am- } \\
\text { bos grupos (aunque no hubo diferencias } \\
\text { estadísticamente significativas al compa- } \\
\text { rarlos). }\end{array}$ \\
\hline $\begin{array}{l}\text { Mitchell y } \\
\mathrm{col}^{14}, 2005\end{array}$ & $\begin{array}{l}\text { Ensayo clínico } \\
\text { aleatorizado. }\end{array}$ & $\begin{array}{l}160 \text { pacientes, } 80 \\
\text { cuya intervención } \\
\text { sería en casa. Y } 80 \\
\text { con intervención en } \\
\text { el hospital }\end{array}$ & $\begin{array}{l}\text { Averiguar la efectividad pre y posto- } \\
\text { peratoria de la fisioterapia en casa } \\
\text { tras prótesis de rodilla. Medidas } \\
\text { tomadas preoperatoriamente y a } \\
\text { las } 12 \text { semanas tras la intervención. }\end{array}$ & $\begin{array}{l}\text { Medidas de resultados: cuestionario de } \\
\text { calidad de vida, WOMAC, satisfacción del } \\
\text { paciente, recursos utilizados. } \\
\text { No hubo diferencias en la primera medida } \\
\text { tomada. El grupo tratado en casa necesitó } \\
\text { más sesiones que el grupo del hospital. } \\
\text { No hubo diferencias en los costes de la in- } \\
\text { tervención, aunque sí fue más cara la RHBc } \\
\text { en casa, con una diferencia de } 136,5 \text { libras. } \\
\text { Los pacientes estuvieron igual de satisfe- } \\
\text { chos, sin embargo el grupo de casa volvería } \\
\text { a solicitarlo allí. }\end{array}$ \\
\hline $\begin{array}{l}\text { Brunenberg } \\
\text { y coll }^{43} \\
2005\end{array}$ & $\begin{array}{l}\text { Estudio Antes- } \\
\text { Después (Before-after } \\
\text { trial). Selección } \\
\text { consecutiva. }\end{array}$ & $\begin{array}{l}160 \text { participantes } \\
\text { Cadera: } \\
\text { Experimental } \mathrm{n}=48 \\
\text { Cuidados estandar } \\
\mathrm{n}=50 \\
\text { Rodilla: } \\
\text { Experimental } \mathrm{n}=30 \\
\text { Cuidados estandar } \\
\mathrm{n}=32\end{array}$ & $\begin{array}{l}\text { Determinar el coste-efectividad de } \\
\text { un programa para pacientes (Joing } \\
\text { Recovery Programe) consistente en } \\
\text { cuidados estandarizados con edu- } \\
\text { cación al paciente y rehabilitación } \\
\text { en grupo, comparado con un grupo } \\
\text { de cuidados estandarizados sin } \\
\text { educación ni RHB'. } \\
\text { Medidas en el preoperatorio, a las } \\
7,12,26,52 \text { semanas del postope- } \\
\text { ratorio. }\end{array}$ & $\begin{array}{l}\text { Se miden el nivel de funcionalidad (Ameri- } \\
\text { can Knee Society Store) y calidad de vida; } \\
\text { media de los costes por paciente. } \\
\text { El nivel de funcionalidad y la calidad de } \\
\text { vida fue más alta en los pacientes tratados } \\
\text { en el grupo con Join Recovery Programe, } \\
\text { incluso más allá de un año. El objetivo más } \\
\text { importante del programa fue reducir la per- } \\
\text { manencia de los pacientes en el hospital. }\end{array}$ \\
\hline $\begin{array}{l}\text { Moffet y } \\
\operatorname{col}^{38}, 2004\end{array}$ & $\begin{array}{l}\text { Ensayo clínico } \\
\text { aleatorizado (buena } \\
\text { calidad } 7 / 10) \text {. } \\
\text { Selección aleatoria, } \\
\text { doble ciego. }\end{array}$ & $\begin{array}{l}77 \text { personas } \\
\text { osteoartrosis de } \\
\text { rodilla. } 2 \text { meses tras } \\
\text { la intervención. } \\
\text { Grupo experimental: } \\
38 \text { pacientes que } \\
\text { recibieron RHBc } \\
\text { supervisada y } \\
\text { ejercicios en casa } \\
\text { entre } 2-4 \text { meses. } \\
\text { Grupo control: } 39 \text { con } \\
\text { cuidados estándar. }\end{array}$ & $\begin{array}{l}\text { Evaluar la efectividad de una reha- } \\
\text { bilitación intensiva y funcional para } \\
\text { alcanzar funcionalidad y calidad } \\
\text { de vida tras artroplastia de rodilla. } \\
\text { Evaluados a los } 2 \text { meses de la inter- } \\
\text { vención y de } 2 \text { a } 8 \text { meses más tarde. }\end{array}$ & $\begin{array}{l}\text { Las medidas de resultado han sido 6-minute } \\
\text { walk test, la escala WOMAC, y SF-36. } \\
\text { Los resultados indican que los sujetos con } \\
\text { el tratamiento de rehabilitación caminaron } \\
\text { durante más tiempo largas distancias. Tu- } \\
\text { vieron menos dolor y mayor funcionalidad } \\
\text { para las actividades de la vida diaria. }\end{array}$ \\
\hline
\end{tabular}




\begin{tabular}{|c|c|c|c|c|}
\hline $\begin{array}{l}\text { Autores } \\
\text { y Año }\end{array}$ & Diseño o Método & Participantes & Intervenciones y Seguimiento & Variables medidas y Resultados \\
\hline $\begin{array}{l}\text { Kramer y } \\
\mathrm{col}^{29}, 2003\end{array}$ & $\begin{array}{l}\text { Ensayo clínico } \\
\text { aleatorizado (buena } \\
\text { calidad) }\end{array}$ & $\begin{array}{l}160 \text { pacientes } \\
\text { intervenidos con } \\
\text { artroplastia de } \\
\text { rodilla. } \\
\text { Grupo experimental } \\
\mathrm{N}=80 \\
\text { Control } \\
\mathrm{N}=80\end{array}$ & $\begin{array}{l}\text { Se compara un programa de RHB } \\
\text { guiado por un fisioterapeuta am- } \\
\text { bulatoriamente, hasta las } 12 \text { se- } \\
\text { manas después de la intervención } \\
\text { ( } 2 \text { veces/semana, } 1 \text { hora) con otro } \\
\text { de RHB en casa monitorizada por } \\
\text { llamadas telefónicas periódicas } \\
\text { realizadas por un fisioterapeuta al } \\
\text { menos una vez durante las semanas } \\
2 \text { a la } 6 . \\
\text { Seguimiento prequirúrgico, des- } \\
\text { pués de la intervención a las } 6,12, \\
24 \text { semanas y a las } 52 .\end{array}$ & $\begin{array}{l}\text { Las medidas fueron la: Knee Society Clinical } \\
\text { Rating Scale, WOMAC, calidad de vida, do- } \\
\text { lor, funcionalidad, distancia caminada en } 6 \\
\text { minutos; } \mathrm{n}^{0} \text { de escaleras ascendidas y des- } \\
\text { cendidas en } 30 \text { segundos. Rango de flexión } \\
\text { de la rodilla. } \\
\text { No hubo diferencias estadísticamente sig- } \\
\text { nificativas entre ambos grupos aunque se } \\
\text { necesitan más estudios para determinar los } \\
\text { pacientes que están más satisfechos con } \\
\text { los beneficios obtenidos. }\end{array}$ \\
\hline $\begin{array}{l}\text { Frost y } \\
\mathrm{col}^{37}, 2002\end{array}$ & $\begin{array}{l}\text { Ensayo clínico } \\
\text { aleatorizado. Buena } \\
\text { calidad }\end{array}$ & $\begin{array}{l}47 \text { pacientes con } \\
\text { prótesis unilateral de } \\
\text { rodilla. } \\
24 \text { pacientes con un } \\
\text { programa tradicional } \\
\text { de ejercicios en casa; } \\
23 \text { pacientes con } \\
\text { ejercicios funcionales } \\
\text { en casa tras el alta. }\end{array}$ & $\begin{array}{l}\text { Comparar estos dos tipos de ejer- } \\
\text { cicios para prótesis de rodilla en } \\
\text { casa, repercusión sobre la movili- } \\
\text { dad y funcionalidad. } \\
\text { Seguimiento a los } 3,6 \text { y } 12 \text { meses } \\
\text { después de la intervención. }\end{array}$ & $\begin{array}{l}\text { Las medidas fueron balance articular (go- } \\
\text { niómetro), escala del dolor, test para la } \\
\text { marcha. } \\
\text { Hubo mejoras en la movilidad, extensión y } \\
\text { dolor al año tras la intervención en ambos } \\
\text { grupos. } \\
\text { No hubo diferencias estadísticamente signi- } \\
\text { ficativas entre los dos grupos. }\end{array}$ \\
\hline $\begin{array}{l}\text { Nelly y } \\
\operatorname{col}^{44}, 2000\end{array}$ & $\begin{array}{l}\text { Estudio Descriptivo } \\
\text { Comparativo }\end{array}$ & $\begin{array}{l}100 \text { Sujetos. } \\
51 \text { de cadera y } 49 \text { de } \\
\text { rodilla }\end{array}$ & $\begin{array}{l}\text { Examinar los resultados clínicos y } \\
\text { económicos en prótesis: descarga } \\
\text { a casa o a una unidad de procesos } \\
\text { subagudos. } \\
\text { Grupo A: sujetos que son derivados } \\
\text { a casa con fisioterapia (durante } 21 \\
\text { días, } 3 \text { sesiones/semana, } 1 \text { hora) } \\
\text { Grupo B: fisioterapia en la unidad } \\
\text { de subagudos, } 2 \text { sesiones de fisio- } \\
\text { terapia al día, } 7 \text { días a la semana } \\
\text { durante } 10 \text { días. } \\
\text { Las medidas se tomaron al mes y a } \\
\text { los } 3 \text { meses. }\end{array}$ & $\begin{array}{l}\text { Las medidas fueron el Joint Ranking Ques- } \\
\text { tionnaire, comportamiento subjetivo del } \\
\text { sujeto; dolor, medicamentos, deambulación, } \\
\text { AVD, costes y permanencia de la estancia. } \\
\text { Los resultados fueron mejores a los } 3 \text { meses } \\
\text { que al mes. } \\
\text { No hubo diferencias en los resultados } \\
\text { aunque en el hospital recibiesen más tra- } \\
\text { tamiento. } \\
\text { El coste fue mayor para los pacientes del } \\
\text { hospital aunque no fue estadísticamente } \\
\text { significativo. }\end{array}$ \\
\hline
\end{tabular}

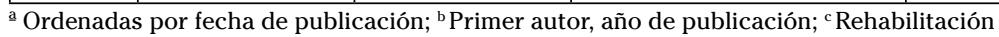

${ }^{\mathrm{d}}$ Artroplastia Total de Rodilla; ${ }^{\mathrm{e}}$ Movimiento Pasivo Continuo

El nivel de actividad o funcionalidad del paciente, lo tuvieron en cuenta seis estudios con medidas como la de la American Knee Society Store ${ }^{43}$ entre otras ${ }^{11,16,26,29,36}$.

Hacen alusión al índice de Barthel o actividades para la vida diaria tres de los estudios $^{16,42,44}$. La escala WOMAC que mide dolor, rigidez y funcionalidad es utilizada en siete ${ }^{11,14,17,26,29,35,38}$ de las investigaciones.

El rango articular de la rodilla fue medido a través del goniómetro, o escalas como el Oxford Knee Store o el Barlet patellar sto$r e$, en nueve estudios ${ }^{11,15,16,24,29,37,41,40,44}$ donde se hacía alusión al movimiento pasivo o activo, a la flexión y la extensión de la rodilla.

La fuerza muscular, sobre todo del cuádriceps, fue tomada en consideración en tres estudios ${ }^{24,41,42}$. Para la marcha, nueve de los estudios usaron alguna forma de medirla ${ }^{16,37,42,44}$, incluyendo velocidad ${ }^{29}$ (número de escaleras ascendidas y descendidas en 30 segundos), distancia ${ }^{15} \mathrm{o}$ test $^{29,38}$ (6 minutes walk test) y dispositivos de ayuda ${ }^{15,24}$.

La calidad de vida se midió sobre todo con el cuestionario SF-36 ${ }^{14,16,26,29,35,38,42,43}$ también con el SF-12 $2^{40}$ y Lavernia y col $^{17}$ con un cuestionario de bienestar. La satisfacción de los pacientes fue una variable que consideraron tres de las investigaciones ${ }^{11,14,35}$.

También fue tenida en cuenta la duración de la estancia hospitalaria, los reingresos o readmisión, las tasas de mortalidad, utilización de servicios y carga del cuida- 
dor o soporte social en cinco de los estudios revisados ${ }^{25,26,36,41,44}$.

El coste o coste-efectividad de los tratamientos aplicados, fue un tema tratado en siete trabajos a través de diversas medidas como los gastos quirúrgicos directos e indirectos de personal y cuidados en casa ${ }^{17}$, además de los recursos utilizados ${ }^{14,25,35}$, ${ }_{36,43,44}$. La idea de que el coste es menor en el domicilio que asistiendo a rehabilitación ambulatoriamente ha sido apoyada por la mayoría de los autores ${ }^{17,35,44}$. Tan sólo Michell y col$^{14}$ afirman que la fisioterapia en casa fue más cara que la realizada en el hospital, con una diferencia de 136,5 libras, aunque igual de efectiva.

Brunenberg y col $^{43}$ también estudian el coste-efectividad, ya que la estancia media en el hospital de los pacientes, que fueron tratados posteriormente con rehabilitación fue inferior a la de los pacientes que eran derivados a casa habitualmente (la diferencia fue de 4,1 días en cadera y 6,9 días en rodilla, con diferencias estadísticamente significativas). Los costes medios totales fueron superiores en la atención habitual que cuando se derivaban al programa de rehabilitación.

Tres estudios tuvieron en cuenta la duración del tratamiento y cantidad de las sesiones $^{11,15,25}$.

Las complicaciones sólo fueron valoradas en el estudio de Iyengar y $\mathrm{col}^{25}$. No se han destacado en ninguno de los estudios efectos adversos atribuibles a la rehabilitación.

\section{Efectividad de la rehabilitación o fisioterapia domiciliaria comparada con otras intervenciones}

Cinco autores han abordado directamente la comparación de la rehabilitación o fisioterapia domiciliaria con la ambulatoria, sin especificar otro tipo de aplicaciones o tratamientos. Mahomed y col ${ }^{35}$ afirman que no hubo diferencias estadísticamente significativas en funcionalidad (WOMAC), calidad de vida (SF-36) o satisfacción entre los dos grupos a los tres o a los 12 meses; también las complicaciones fueron las mismas, pero el número de sesiones ambula- torias fue superior a las realizadas en el domicilio y por lo tanto también los costes asociados fueron mayores. Iyengar y $\mathrm{col}^{25}$ además añaden que el programa de rehabilitación domiciliaria reduce la estancia hospitalaria en relación con la rehabilitación convencional, sin observarse mayores complicaciones ni reingresos pero no revela una síntesis de los costes y beneficios del servicio ofrecido.

Tampoco Lavernia y coll $^{17}$ encuentran diferencias estadísticas aunque las mejorías en la función y el dolor se producían tras el tratamiento en ambos grupos y en calidad de vida los pacientes atendidos en casa obtuvieron mayor puntuación.

Mitchell y $\mathrm{col}^{14}$ respaldan la idea de que la fisioterapia en casa fue tan efectiva y aceptable como la recibida ambulatoriamente, aunque la media de sesiones fue mayor en el domicilio, y el tiempo de espera para realizar la fisioterapia en el hospital (18 días) superior a los tres días de los que comenzaban el tratamiento en casa. Aunque los pacientes estuvieron igual de satisfechos en ambos grupos, el grupo de casa reiteró que volvería a solicitar la rehabilitación allí.

Con respecto a la fisioterapia preoperatoria domiciliaria, no mejoró los resultados percibidos en salud. Collins y col $^{15}$ hallan que el número de visitas domiciliarias de fisioterapia disminuyó después de la implantación de la prótesis y también las sesiones fueron mejor aprovechadas (más eficientes y funcionales) que las sesiones preoperatorias; aumentando además la tendencia a caminar mayores distancias en los pacientes.

Cuando se combina fisioterapia domiciliaria con movimiento pasivo continuo $\mathrm{o}^{41} \mathrm{se}$ producen aumentos pequeños en la amplitud de la flexión activa de la rodilla, disminuye la duración de la estancia hospitalaria y se reduce el riesgo de manipulación tras artroplastia; pero estos beneficios potenciales deberían analizarse con cautela frente a los inconvenientes y gastos que implica.

En relación a la rehabilitación domiciliaria, definida por un folleto de ejercicios destinados a pacientes con prótesis de rodilla y a realizar en casa, diversos autores 
como $\mathrm{Mozo}^{16}$ (en 2008) la han comparado con la rehabilitación ambulatoria. Este autor concluyó que ambas ofrecían resultados similares. Mockford y $\mathrm{col}^{40}$ en cambio, encontraron que los pacientes que recibieron tratamiento fisioterápico ambulatorio obtuvieron mayor rango articular, pero la diferencia con respecto a los que la realizaban en casa, no era estadísticamente significativa.

La revisión sistemática de Minns y col $^{42}$, ofrece soporte al uso de ejercicios funcionales fisioterapéuticos después del alta, comparados con los tradicionales ejercicios ofrecidos para realizar en casa y que eran enseñados durante la hospitalización (estiramientos y ejercicios isométricos de cuádriceps). Hubo significación moderada a los 3 o 4 meses del postoperatorio, donde se aumentó el rango articular y la calidad de vida; pero no se observó que los beneficios persistiesen tras un año de seguimiento.

Brunenberg y col $^{43}$ vienen a confirmar también estos hallazgos al comparar el grupo que realizó un programa de fisioterapia y comprobar que el rango articular, la funcionalidad y la calidad de vida fue más alta, con diferencias estadísticamente significativas. Aunque los datos fueron superiores más allá de un año, no hubo diferencias estadísticas, pero se logró el objetivo más importante del programa, que era reducir la permanencia del paciente en el hospital.

Moffet y col${ }^{38}$, comparan los ejercicios en casa (cuidados estándar) con un programa de rehabilitación intensiva y funcional supervisada más ejercicios llevados a cabo en casa, a los dos meses de la intervención. Todos los pacientes asignados a los cuidados estándar no realizaron el mismo tratamiento, ya que una tercera parte recibieron una media de 7 sesiones de fisioterapia domiciliaria, mientras otros sólo los ejercicios encomendados. El efecto del tratamiento de la rehabilitación ambulatoria o funcional fue modesto, pero consistente (estadísticamente significativo); los pacientes tenían menos dolor, rigidez y dificultad para realizar las actividades de la vida diaria, caminando distancias más largas, aunque no hubo diferencias en el cuestionario de calidad de vida, salvo en el rol físico donde las puntuaciones fueron mayores. Este programa fue más efectivo para la mejora de la funcionalidad a corto y a medio plazo, pero no a largo plazo (a los 12 meses). Anteriormente, Frost y col${ }^{37}$ en el año 2002, habían demostrado también mejoras en la movilidad, extensión y dolor al año tras la intervención fisioterápica en ambos grupos, pero sin hallar tampoco diferencias estadísticamente significativas; aunque los pacientes que no perseguían la funcionalidad con sus ejercicios fueron perdiendo la motivación durante la realización de las sesiones.

Otra variedad de tratamiento fueron los ejercicios de casa controlados telefónicamente por un fisioterapeuta (con cinco llamadas) y la revisión por un cirujano, comparados con un programa de rehabilitación ambulatorio durante 12 semanas en las que recibieron una media de 15 sesiones. Kramer y col$^{29}$ afirman que se produjeron similares resultados en ambos grupos medidos a las 52 semanas tras la intervención, pero algunos pacientes tuvieron que ser cambiados durante el seguimiento del programa en casa a la rehabilitación ambulatoria debido al fallo en la progresión del tratamiento (dolor, actitud y motivación). También fueron derivados los pacientes identificados con potenciales complicaciones.

Se ha estudiado la rehabilitación domiciliaria comparándola con un servicio subagudo de rehabilitación previo a la derivación de fisioterapia domiciliaria ${ }^{24,44}$. Estos programas de fisioterapia domiciliaria varían de un lugar a otro y entre países, desde 3-5 veces a la semana ${ }^{24}$; hasta tratamientos de tan sólo una hora a la semana de fisioterapia y la supervisión por una enfermera de los cuidados ${ }^{44}$. Pero los resultados de los estudios han sido equivalentes, ambos programas de rehabilitación ofrecieron resultados clínicos similares, aunque los pacientes que estuvieron en el servicio de rehabilitación del hospital recibieron más tratamiento ${ }^{24}$ (una media de 12,4 días más) y los costes fueron por lo tanto sustancialmente diferentes ${ }^{44}$ (mayor para los pacientes del hospital). 
El programa Groningen Orthopaedic Exit Strategy enfatiza la continuación de la fisioterapia domiciliaria motivada a través de vídeos, cartas y consultas telefónicas en comparación con un grupo que sólo recibió instrucciones durante el ingreso y una llamada tras el alta, pero no se hallaron diferencias estadísticamente significativas entre los grupos experimental y control respecto al efecto del tratamiento ni a las 6 ni a las 26 semanas. Únicamente se encontraron diferencias en la percepción del soporte social a favor del grupo control.

En relación a la atención multidisciplinaria domiciliaria, Khan y col$^{36}$ afirmaron con su revisión sistemática que mejoró la ganancia funcional, la calidad de vida y redujo la estancia hospitalaria, a medio plazo (6 meses) aunque ninguno de sus ensayos abordó el largo plazo.

\section{CONSIDERACIONES FINALES}

La proliferación de estudios en los últimos años en relación a la rehabilitación o fisioterapia domiciliaria se ha destacado, ya que en esta revisión más de la mitad de los estudios (8 de los 15) se han gestado entre los años 2007 y 2008, así como las tres revisiones sistemáticas halladas. Se pone de manifiesto el creciente interés que han manifestado las políticas sanitarias en los distintos países al apostar por tratamientos que se lleven a cabo fuera de las instituciones hospitalarias, aunque aún no existe consenso sobre la definición de las técnicas o medios para realizar estos tratamientos en el domicilio.

Se ha encontrado que incluso en ciertos estudios se enmascara la ausencia de tratamiento rehabilitador o fisioterapéutico, describiendo que el paciente realizó rehabilitación domiciliaria o en casa, cuando sólo recibe algún consejo, recomendación o un folleto explicativo con ejercicios generales para su recuperación tras la intervención.

En conjunto, los estudios incluidos aportan un cuerpo de pruebas que afirman que la rehabilitación domiciliaria puede mejorar la experiencia de las personas tras las prótesis en cuanto a la actividad funcio- nal y participación, pero existen pruebas insuficientes para comparar la efectividad de los ámbitos de atención domiciliaria y hospitalaria sobre los resultados de la rehabilitación en prótesis de rodilla (la descripción y especificación del ámbito no ha estado adecuadamente descrita en todos los estudios; los grupos de intervención o control no han sido comparables, y son pocos los estudios que incorporan el coste como un atributo más de los que definen el "perfil" de un tratamiento).

Las limitaciones de este trabajo están sujetas sobre todo a las propias limitaciones de los artículos incluidos. La mayoría de los ensayos y revisiones (18) que contaron en total con 4.996 participantes, fueron pequeños y todos presentaron limitaciones metodológicas, como fue el seguimiento inadecuado. En comparación con los ensayos clínicos aleatorizados, con menos de 100 participantes de media ${ }^{45}$, las revisiones contienen considerablemente más información ${ }^{46}$ y los estudios que han sido recogidos aportan información relevante sobre el panorama actual en distintos países de la geografía mundial y sus experiencias en relación a las políticas sanitarias que aplican.

Este procedimiento de revisión exhaustiva ha aplicado estrategias científicas para limitar los sesgos en el proceso de recopilación de información, realizando la valoración crítica y síntesis de los estudios relevantes sobre el tema. Los datos han revelado que eran demasiado heterogéneos para realizar un meta-análisis, y ha sido imposible aplicar métodos estadísticos, entre otras causas por las diferencias entre los diseños de los estudios (algunos no especifican cómo son asignados los pacientes a cada grupo $)^{16}$, las distintas intervenciones realizadas sobre el grupo experimental y control, o el uso de diversas medidas de resultado en distintos puntos temporales de la evolución del paciente. Khan y coll $^{36}$ en 2008 tampoco logra este objetivo ya que la heterogeneidad y baja calidad de los ensayos incluidos no permitieron un análisis agrupado.

Tanto el sistema sanitario español, como el resto de países, necesitan profundizar en investigación para identificar 
los instrumentos de racionamiento, que siendo eficientes y equitativos, ofrezcan mayores probabilidades de mejorar las intervenciones en salud en una población que demanda mayores procesos y procedimientos asistenciales ${ }^{47}$. Informar sobre el coste-efectividad de los tratamientos permitiría avanzar en la toma de conciencia por parte del clínico y del paciente, haciéndonos conscientes del precio en términos de coste de oportunidad, que la sociedad paga para la obtención de los beneficios de salud derivados ${ }^{48}$.

Conforme las investigaciones en relación a este tema sean más rigurosas, será necesario realizar nuevas revisiones sobre las intervenciones que hayan cambiado o mejorado con el tiempo. Muchas guías clínicas utilizan los resultados de una revisión sistemática como punto de partida; o las agencias de financiación exigen a veces evidencia en forma de revisiones como ésta para patrocinar una nueva investigación clínica ${ }^{49,20}$.

Aunque no se haya establecido aún la base de pruebas normalizadas para demostrar la efectividad de la rehabilitación y fisioterapia domiciliaria en las personas con reemplazo articular de rodilla comparada con otro tipo de intervención, esta revisión brinda un apoyo a la recomendación de evaluar a estos pacientes a fin de conocer la necesidad de rehabilitación apropiada para maximizar su capacidad funcional independiente.

Se puede afirmar que existe insuficiente evidencia científica para comparar esta efectividad en los distintos servicios, ya que se necesitan estudios bien diseñados para determinar el valor de los ejercicios y técnicas empleadas en los diversos ámbitos de aplicación, con seguimientos adecuados. La mayoría de las investigaciones que se han revisado afirman que la fisioterapia domiciliaria acelera la consecución de logros funcionales a corto y medio plazo, conllevando estancias hospitalarias más breves y reducción de costes con respecto a la rehabilitación ambulatoria. La fisioterapia funcional postoperatoria inmediata resulta beneficiosa para el rango de movimiento, la función y calidad de vida.
Las conclusiones de este artículo desearían generar la inquietud para que se realicen investigaciones experimentales, bien diseñadas, que son tan necesarias a nivel nacional e internacional en los momentos que vivimos de escasez de recursos económicos. A medida que aumenta la presión sobre los sistemas de salud, resulta esencial planificar una asistencia sanitaria asequible y apropiada, debiéndose justificar el uso de los procedimientos de tratamiento más costosos.

\section{BIBLIOGRAFÍA}

1. Gómez J. Calidad de vida relacionada con la salud. La punta del iceberg del complejo abordaje de los ancianos muy mayores. Med Clín (Barc) 2010; 135:162-164.

2. Consejería de Salud. Artrosis de rodilla y cadera: Proceso Asistencial Integrado. Sevilla, España: Junta de Andalucía; 2004, p122.

3. Estudio EPISER 2000. Prevalencia e impacto de las enfermedades reumáticas en la población adulta española. Madrid: MSD y Sociedad Española de Reumatología; 2001, p167.

4. Pendleton A, Arden N, Dougados M. EUlaR recommendations for the management of knee osteoarthritis. Ann Rheum Dis 2000; 59: 936944.

5. ACKROYD CE. Medial compartment arthroplasty of the knee. J Bone Joint Surg 2003; 85 Suppl. B: 937-942.

6. RiBERA JM. Epidemiología de la enfermedad osteoarticular en la persona mayor. JANO 2005; 64: 28-23.

7. Batlle-Gualda E. Estudio ArtRoCad: evaluación de la utilización de los recursos sanitarios y la repercusión socioeconómica de la artrosis de rodilla y cadera. Presentación de resultados preliminares. Rev Esp Reumatol 2005; 32: 22-27.

8. Pagés E, Iborra J, Rodríguez S, Jou N, Cuxart A. Prótesis total de rodilla. Estudio de los factores determinantes del alta hospitalaria en rehabilitación. Rehabilitación 2002; 36: 202207.

9. Riu M, Muniesa J, Leal J, Cots F, Cáceres E, CasTELLS X. Evaluación del impacto asistencial de la puesta en funcionamiento de una unidad funcional de artroplastia de rodilla. Gac Sanit. 2009; 23: 444-447.

10. Renkawitz T, Rieder T, Handel M, koller M, DresCHER J, BonnlaENDER G et al. Comparison of two 
accelerated clinical pathways - after total knee replacement how fast can we really go Clin Rehabil 2010; 24: 230-239.

11. Lenssen AF, Van Steyn JA, Crijins HF, Waltjé MH, Roox GM, GEESINK JT et al. Effectiveness of prolonged use of continuous passive motion (CPM), as an adjunct to physiotherapy, after total knee arthroplasty. BMC Musculoskelet Disord 2008; 9: 60.

12. Ethgen O, Bruyere O, Richy F, Dardennes C, ReGINSTER JY. Health-related quality of life in total hip and total knee arthroplasty. A qualitative and systematic review of the literature. J Bone Joint Surg Am 2004; 86: 963-974.

13. Kauppila A, HämäläInen M, MäKI-HeIKKIlä P, SINtonen H, LepPilahti J, ARokoski J. Multidisciplinary rehabilitation after primary total knee arthroplasty: a randomized controlled study of its effects on functional capacity and quality of life. Clin Rehabil 2010; 24: 398-411.

14. Mitchell C, Walker J, Walters S, Morgan A, Binns T, MAthers N. Costs and effectiveness of preand post-operative home physiotherapy for total knee replacement: randomized controlled trial. J Eval Clin Pract 2005; 11: 283-292.

15. Collins T, Herness J, Martenas J, Roberson A. Medicare prospective payment before and after implementation. A review of visits and Physical Performance among Medicare Home Health patients after total knee replacements. Home Healthc Nurse 2007; 25: 401407.

16. Mozo AP, Moreno JA, Plazas N, Manjón-Cabeza JM, Ruiz D, CÁtedra EV. Comparación de dos programas de rehabilitación tras artroplastia total de rodilla. Trauma Fundación MAPFRE 2008; 19: 218-224.

17. Lavernia C, D'Apuzzo M, Hernández V, Lee D, RossI M. Postdischarge costs in arthroplasty surgery. J Arthroplasty 2006; 21 Supl. 2: 144-150.

18. IMSERSO. Cuidado a las personas mayores en los hogares españoles. Madrid: Ministerio de Trabajo y Asuntos Sociales. 2005

19. López-Liria R, Padilla D, Catalán D, Arrebola C, Garrido P, Martínez MC. Análisis de la actividad en las unidades móviles de rehabilitación-fisioterapia en atención primaria. Aten Primaria 2010; 42: 278-283.

20. López-Liria R, Padilla-Góngora D, Catalán-Matamoros D, Rocamora-Pérez P, Martínez-Cortés MC y Rodríguez-Martín C. Análisis de las patologías con mayor prevalencia en las Unidades Móviles de Rehabilitación y Fisioterapia de la provincia de Almería. Gac Sanitaria 2011 Dec 2. PMID: 22138280.
21. Clarke M, Oxman AD (eds.). Manual del revisor cochrane 4.1.6 (actualización enero 2003). En: The Cochrane Library, Número 1, Oxford. 2005. Actualización Octubre de 2008. Disponible en: http://www.cenetec.salud.gob.mx/ descargas/presentacionesforo2007/17sep/ evaluacion/carlos_jimenez_gutierrez.pdf

22. Galea MP, Levinger P, Lythgo N, Cimoli C, Weller $\mathrm{R}$, Tully $\mathrm{E}$ et al. A targeted home- and centerbased exercise program for people after total hip replacement: a randomized clinical trial. Arch Phys Med Rehabil 2008; 89:14421447. Epub 2008 Jun 30.

23. Sigurdsson E, Siggeirsdottir K, Jonsson H, GudnaSON V, MatThiasson T, Jonsson BY. Early discharge and home intervention reduces unit costs after total hip replacement: results of a cost analysis in a randomized study. Int $\mathrm{J}$ Health Care Finance Econ 2008; 8:181-192.

24. Chimenti CE, Ingersoll G. Comparison of home health care physical therapy outcomes following total knee replacement with and without subacute rehabilitation. J. Geriatr Phys Ther 2007; 30: 102-108.

25. Iyengar KP, NadKarni JB, Ivanovic N, Mahale A. Targeted early rehabilitation at home after total hip and knee joint replacement: does it work? Disabil Rehabil 2007; 29: 495-502.

26. Van den AkKer-Scheek I, ZiJlstra W, Groothoff J, Van Horn J, Bulstra S, Stevens M. Groningen orthopaedic exit strategy: validation of a support program after total hip or knee arthroplasty. Patient Educ Couns 2007; 65: 171-179.

27. Polzien G. Care after hip replacement. Home Healthc Nurse 2006; 24: 420-422.

28. De Pablo P, Losina E, Phillips CB, Fossel AH, MAHOMED N, Lingard EA et al. Determinants of discharge destination following elective total hip replacement. Arthritis Rheum 2004; 51: 1009-1017.

29. Kramer JF, Speechley M, Bourne R, Rorabeck C, VAZ M. Comparison of clinic- and homebased rehabilitation programmes after total knee arthroplasty. Clin Orthop Relat Res 2003; 410: 225-234.

30. Loft, M. Mc William, C. Ward-Griffin, C. Patient empowerment after total hip and knee replacement. Orthop Nurs 2003; 22: 42-47.

31. Engh CA, Culpepper WJ, Charette PA, Brown R. Patient self-testing of protrombin time after hip arthroplasty. J South Orthop Assoc 2001; 10:140-146.

32. Heaton J, Mc Murria RM, Sloper T, Nettleton S. Rehabilitation and total hip replacement: patients perspectivas on privisión. Int J Rehabil Res 2000; 23: 253-259. 
33. Kane RL, Chen Q, Finch M, Blewett L, Burns R, Moskowitz M. The optimal outcomes of posthospital care under Medicare. Health Serv Res 2000; 35: 615-661.

34. Woo T, Bramwell M, Greenwood B, Gow S, AcKERMAN-RAINVILLE R, CORRADETTI P, et al. Integrated systems to reduce length of stay for knee and hip joint replacement surgeries. Healthc Manage Forum 2000 Fall; 13: 60-62.

35. Mahomed NN, Davis AM, Hawker G, Badley E, DAVEY JR, SYED KA et al. Inpatient compared with home-based rehabilitation following primary unilateral total hip or knee replacement: a randomized controlled trial. J Bone Joint Surg Am 2008; 90: 1673-1680.

36. Khan F, NG L, Gonzalez S, Hale T, Turner-Stokes L. Multidisciplinary rehabilitation programmes following joint replacement at the hip and knee in chronic arthropathy. Cochrane Database Syst Rev 2008; CD004957.

37. Frost H, Lamb SE, Robertson S. A randomized controlled trial of exercise to improve mobility and function after elective knee arthroplasty. Feasibility, results and methodological difficulties. Clin Rehabil 2002; 16: 200-209.

38. Moffet H, Collect JP, Shapiro SH, Paradis G, MARQuis F, Roy L. Effectiveness of intensive rehabilitation on functional ability and quality of life after first total knee arthroplasty: a single-blind randomized controlled trial. Arch Phys Med Rehabil 2004; 85: 546-556.

39. Davies DM, Johnston DW, Beaupre LA, LiER DA. Effect of adjunctive range of motion therapy after primary total knee arthroplasty on the use of health services after hospital discharge. Can J Surg 2003; 46: 30-36.

40. Mockford B, Thompson N, Humphreys P, BeverLAND D. Does a standar outpatient physiotherapy regime improve the range of knee motion after primary total knee arthroplasty? J Arthroplasty 2008; 23:1110-1114.
41. Milne S, Brosseau L, Robinson V, Noel MJ, Davis $\mathrm{J}$, Drouin $\mathrm{H}$ et al. Movimiento pasivo continuo posterior a la artroplastia total de rodilla. En: Biblioteca Cochrane Plus. 2007. Numero 4. Oxford: Update Software Ltd. Disponible en: http://sss.up-date-software.com.

42. Minns CJ, Barker KL, Dewey M, Sackley CM. Effectiveness of physiotherapy exercise after knee arthroplasty for osteoarthritis: systematic review and meta-analyses of randomized controlled trials. BMJ 2007; 335: 812820 .

43. Brunenberg DJ, Van Steyn M, Sluimer JC, BekeBREDE L, Bulstra S, JoORE MA. Joint recovery programme versus usual care. An economic evaluation of a clinical pathway for joint replacement surgery. Med Care 2005; 43: 10181026.

44. Kelly M, Tilbury M, Ackerman R. Evaluation of fiscal and treatment outcomes in major joint replacement. Outcomes Manag Nurs Pract 2000; 4: 46-50.

45. Altman DG, Simera I, Hoey J, Moher D, Schulz K. EQUATOR: Reporting guidelines for health research. Lancet 2008; 371: 1149-1150.

46. Hopewell S, Dutton S, Yu LM, Chan AW, Altman DG. The quality of reports of randomised trials in 2000 and 2006: comparative study of articles indexed in Pubmed. BMJ 2010; 340: c723.

47. Rodríguez-Monguió R, Antoñanzas F. Healthcare rationing in Spain. Framework, descriptive analysis and consequences. Pharmacoeconomics 2006; 24: 537-548.

48. Eccles M, Mason J. How to develop cost conscious guidelines. Health Technol Assess 2001; 5: 1-69.

49. Moher D, Liberati A. Revisiones sistemáticas y metaanálisis: la responsabilidad de los autores, revisores, editores y patrocinadores. Med Clin (Barc) 2010; 135: 505-506. 
